\title{
Income inequalities in multimorbidity prevalence in Ontario, Canada: a decomposition analysis of linked survey and health administrative data
}

\author{
Luke Mondor ${ }^{1,2}$, Deborah Cohen ${ }^{2,3}$, Anum Irfan Khan ${ }^{2,4}$ and Walter P. Wodchis ${\text { i, } 2,4,5^{*}}^{-}$
}

\begin{abstract}
Background: The burden of multimorbidity is a growing clinical and health system problem that is known to be associated with socioeconomic status, yet our understanding of the underlying determinants of inequalities in multimorbidity and longitudinal trends in measured disparities remains limited.

Methods: We included all adult respondents from four cycles of the Canadian Community Health Survey (CCHS) (between 2005 to 2011/12), linked at the individual-level to health administrative data in Ontario, Canada (pooled $n=113,627$ ). Multimorbidity was defined at each survey response as having $\geq 2$ (of 17) high impact chronic conditions, based on claims data. Using a decomposition method of the Erreygers-corrected concentration index $\left(C_{\text {Erreygers }}\right)$, we measured household income inequality and the contribution of the key determinants of multimorbidity (including socio-demographic, socio-economic, lifestyle and health system factors) to these disparities. Differences over time are described. We tested for statistically significant changes to measured inequality using the slope index (SII) and relative index of inequality (RII) with a 2-way interaction on pooled data.
\end{abstract}

Results: Multimorbidity prevalence in $2011 / 12$ was $33.5 \%$ and the $C_{\text {Erreygers }}$ was -0.085 (Cl: -0.108 to -0.062 ), indicating a greater prevalence among lower income groups. In decomposition analyses, income itself accounted more than twothirds (69\%) of this inequality. Age (21.7\%), marital status (15.2\%) and physical inactivity (10.9\%) followed, and the contribution of these factors increased from baseline (2005 CCHS survey) with the exception of age. Other lifestyle factors, including heavy smoking and obesity, had minimal contribution to measured inequality ( 1.8 and $0.4 \%$ respectively). Tests for trends (SII/RII) across pooled survey data were not statistically significant ( $p=0.443$ and 0.405 , respectively), indicating no change in inequalities in multimorbidity prevalence over the study period.

Conclusions: A pro-rich income gap in multimorbidity has persisted in Ontario from 2005 to 2011/12. These empirical findings suggest that to advance equality in multimorbidity prevalence, policymakers should target chronic disease prevention and control strategies focused on older adults, non-married persons and those that are physically inactive, in addition to addressing income disparities directly.

Keywords: Adult, Chronic disease, Comorbidity, Health status disparities, Ontario/ epidemiology, Prevalence, Socioeconomic factors, Trends, Advance equality in multimorbidity prevalence

\footnotetext{
*Correspondence: walter.wodchis@utoronto.ca

'Institute for Clinical Evaluative Sciences (ICES), G1 062075 Bayview Ave,

Toronto, ON M4N 3M5, Canada

${ }^{2}$ Health System Performance Research Network (HSPRN), 155 College St 4th

Floor, Toronto, ON M5T 3M6, Canada

Full list of author information is available at the end of the article
}

(c) The Author(s). 2018 Open Access This article is distributed under the terms of the Creative Commons Attribution 4.0 International License (http://creativecommons.org/licenses/by/4.0/), which permits unrestricted use, distribution, and reproduction in any medium, provided you give appropriate credit to the original author(s) and the source, provide a link to the Creative Commons license, and indicate if changes were made. The Creative Commons Public Domain Dedication waiver (http://creativecommons.org/publicdomain/zero/1.0/) applies to the data made available in this article, unless otherwise stated. 


\section{Background}

An increasing number of adults in high-income settings have been diagnosed with multiple coexisting chronic conditions [1], also known as multimorbidity. This increased burden has created significant challenges in the effective provision of clinical care and has added pressures on health systems that traditionally provide highly specialized care for a single condition. Extensive research has shown that persons with multimorbidity have a greater risk of functional decline, cognitive decline and early mortality [2-4], are more frequent users of healthcare services, experience greater care fragmentation and longer hospital stays, and also incur higher healthcare costs [5-12].

Multiple studies from high-income settings have shown that multimorbidity is concentrated among persons from lower socio-economic ranks [13-17]. Multimorbidity may develop 10-15 years earlier among young and middle-aged persons in the most (vs. least) deprived areas [18]. While quantifying multimorbidity inequalities may help inform where policy action is needed, less is known about the relative contributions of determinants to socioeconomic disparities in multimorbidity [19]. These data are particularly useful to policymakers for informing future resource allocation and designing targeted interventions. Moreover, few studies have quantified longitudinal trends in measured inequalities in multimorbidity prevalence by income, education, or occupation, despite calls for action by federal agencies and professional organizations in many jurisdictions to reduce health disparities [20, 21].

To address these gaps, we undertook a comprehensive examination of inequalities in multimorbidity prevalence in Ontario, Canada. Our key research objectives were to: 1) quantify household income inequalities in multimorbidity prevalence among adults in Ontario, 2) identify the relative contribution of key determinants of multimorbidity to this inequality including socio-demographic, socio-economic, lifestyle and health system factors; and 3) assess whether these disparities were widening, and whether the drivers contributing to this inequality were changing over time.

\section{Methods}

We used (cross-sectional) survey data and linked health administrative information that is routinely collected in Ontario. The use of this data was authorized under section 45 of Ontario's Personal Health Information Protection Act, which does not require review by a Research Ethics Board. The study is reported according to the RECORD guidelines [22].

\section{Data and setting}

Residents of Ontario, Canada aged 18 years and older that participated in any of four cycles of the Canadian
Community Health Survey (CCHS) - 2005, 2007/08, 2009/10 and 2011/12 - and whose responses were linked to population-based health administrative databases were included in the study. Each survey was analyzed separately, unless otherwise stated (pooled cross-sections for longitudinal analysis).

The CCHS is administered by Statistics Canada to Canadians aged $\geq 12$ years living in private dwellings, and is representative of $98 \%$ of the Canadian population. The CCHS does not include into its sampling frame persons living on Reserves or Crown Lands, institutionalized residents or members of the Canadian Forces. Detailed methodology of the survey and sampling strategy are described elsewhere [23, 24]. Permanent residents of Ontario (2011 population 12.8 million) are covered by a universal health insurance program that covers the costs for most physician and hospital services. Immigrants receive services after a three-month waiting period. Patient encounters with the healthcare system are recorded in administrative databases. Survey and administrative data were linked deterministically at the individual level using unique encoded identifiers and analyzed at the Institute for Clinical Evaluative Sciences (ICES) in Toronto, Ontario. The databases utilized in this study included the Ontario Health Insurance Program claims database (OHIP), Discharge Abstract Database (DAD), Ontario Drug Benefits database (ODB), Registered Persons Database (RPDB), and the Client Agency Program Enrolment database (CAPE) (see: https://datadictionary.ices.on.ca/Applications/DataDiction ary/Default.aspx).

\section{Variables}

We used health administrative data (OHIP, DAD, and ODB data) to determine the prevalence of 17 high-impact chronic conditions for each individual at time of their survey response. Consistent with previous studies of multimorbidity in Ontario [1, 12, 14, 25-27], these conditions were selected based on their economic impact and population burden in the general population. The 17 conditions included: acute myocardial infarction, asthma, cancers, cardiac arrhythmia, chronic coronary syndrome, chronic obstructive pulmonary disorder, congestive heart failure, diabetes, hypertension, mood and anxiety disorders, other mental illnesses (schizophrenia, delusions and other psychoses, personality disorders and substance abuse), osteoarthritis, osteoporosis, renal failure, rheumatoid arthritis and stroke (excluding transient ischemic attack). Where available, validated algorithms were applied to the health administrative data to ascertain cases, including AMI, asthma, COPD, CHD, dementia, diabetes and hypertension [28-34]. As in most other studies [18, 19, 35] we defined multimorbidity from this data as the co-occurrence of 2 or more (of these 17) 
conditions within the same individual, prevalent at the time of survey response.

We used household income from CCHS responses to measure socio-economic status (all respondents are asked: "what is your best estimate of the total income received by all household members, from all sources, before taxes and deductions, in the past 12 months?"). We focused specifically on (household) income as a marker of socioeconomic status to measure inequality because the rich-poor income gap is widening in most countries [36, 37], the strong association relating income to population health is well documented [38], and these gradients are potentially amenable to policy. Because of the sensitive nature of income reporting (resulting in missing or misclassified data), we used the imputed income responses provided by Statistics Canada. This method has been described extensively elsewhere [39]. These data were aggregated to quintiles for analysis and reporting.

Independent variables were selected a priori based on previous literature reporting associations with multimorbidity prevalence from similar high-income jurisdictions $[13,18,40,41]$. Socio-demographic variables included age group (18-34 years, 35-49, 50-64, 65-74 and 75-plus), sex (men and women), rurality (urban, suburban or rural residence - based on the Rurality Index of Ontario [42], from RPDB data), marital status (married vs. divorced, separated, widowed or single), and immigrant status (born in Canada vs. not born in Canada). In addition to income, socio-economic variables (from CCHS data) included the respondent's education level (no post-secondary vs. some post-secondary or higher). Health-related lifestyle characteristics included level of physical activity (active, moderately active, inactive), smoking status (heavy smoker, light smoker, former smoker, non-smoker) and body-mass index (BMI, underweight, normal weight, overweight, obese, following the classifications defined by the World Health Organization [43]). Health system variables included health region of the respondent's residence (i.e., Local Health Integration Network [LHIN]) and enrolment in a primary model of care, which was used a proxy for access to primary care services (Family Health Group [FHG], Family Health Network [FHN], Family Health Organization [FHO], other, and not enrolled [CAPE data]) [44, 45]. Values of survey variables were selected based on the distribution of responses and to reflect meaningful categories. We excluded individuals from analysis if they were missing information (non-response) for any of these variables because there are currently no methods for combining the multiple imputation estimates specific to decomposition analyses.

\section{Statistical analyses}

For each cycle, we transformed income data into cumulative rank probabilities (ridit scores) ranging from 0 (highest income) to 1 (lowest income), with values reflecting the midpoint of the cumulative proportion of the (weighted) population in each income group. Using this variable we quantified inequalities in multimorbidity for each CCHS cycle, first, using the Concentration Index $(C)$ [46]. This relative measure of inequality considers the entire distribution of income, and can be written as:

$$
C=\frac{2}{n \mu} \sum_{i=1}^{n} y_{i} R_{i}-1
$$

where $C$ is the concentration index, $\mu$ is the (weighted) population mean of the outcome (here, multimorbidity prevalence), $y$ is the outcome mean of the $i$ th individual, and $R_{i}$ is the individuals rank in the income distribution. Values range from -1 to +1 , corresponding to a concentration of the outcome (multimorbidity prevalence) among the poorer or wealthier population, respectively, where larger values (approaching -1 or +1 ) indicate greater inequality, and a value of zero indicates equality. Because multimorbidity is dichotomous, we applied the Erreygers correction $\left(\mathrm{C}_{\text {Erreygers }}\right)$, which multiplies $\mathrm{C}$ by 4 times the weighted mean of the health outcome [47]. Standard errors and 95\% confidence intervals (CI) for $\mathrm{C}_{\text {Erreygers }}$ were derived using the methods described by O’Donnell et al. [48].

Wagstaff et al. [49] showed that $\mathrm{C}$ of a continuous health outcome can be decomposed into a set of determinants, a methodology that has since been extended to dichotomous variables [48, 50, 51]. In comparison to traditional analyses, this decomposition method allows for the explanation of the measured health inequality across the entire distribution of socio-economic status. To perform the decomposition, we specified a probit model with marginal effects [48], including all independent variables previously listed in the regression. Income itself is included as a determinant in the decomposition regression to prevent overestimation of the contribution of all other factors to measured inequality [52]. For any determinant to contribute to measured inequality, that determinant must be associated with the health outcome (i.e., have a non-zero regression coefficient) and also be unequally distributed by socio-economic status (i.e., have a non-zero concentration index). A positive (negative) contribution means that that determinant is associated with greater (lower) income inequality in multimorbidity. In addition to the proportional contribution of each determinant is a residual, representing the amount inequality in multimorbidity that cannot be explained by variation of each determinant across the income distribution. Negative residual values are possible, suggesting that determinants entered into the decomposition explain all measured inequality. We present decomposition 
results from the most recent survey, and describe differences in relative contribution of determinants to the baseline cycle.

Multiple sensitivity analyses were performed to assess the robustness of these decomposition analyses. Analyses were repeated using different reference categories in the multivariable probit model [50], performing the decomposition using a logit model approach (i.e., decomposing the natural logarithm of the odds of multimorbidity) [51], decomposing the Wagstaff-corrected C [53], and using a continuous log of household income term in the model (rather than quintiles). For each sensitivity analysis, we assessed any changes in the rank-order of the contribution of determinants measured inequality. Additionally, to ensure the measured inequality was robust to missing data, we re-calculated the C-Erreygers for each survey, including those with missing determinant data.

Lastly, to confirm changes in inequality over the study period, we quantified the relative index of inequality (RII) and slope index of inequality (SII). These measures are recommended for assessing trends in health disparities $[54,55]$. First, we estimated the RII in multimorbidity prevalence within each cycle using a Poisson regression model [56] including covariates for ridit score, age group and sex. The exponential of the ridit score estimate is equivalent to the RII, which can be interpreted as the proportion of multimorbidity prevalence that differs between the highest and lowest incomes. An RII $>1$ is indicative of pro-rich inequality on the relative scale (with larger values suggesting a greater concentration of multimorbidity among the poor). To asses trends, we used the methods outlined by Ernstsen et al. [57] using pooled survey data (with survey-specific ridit scores) and included covariates for ridit score, age group, sex, CCHS cycle and the 2-way ridit"cycle interaction. A positive (negative) and statistically significant interaction term is indicative of increasing (decreasing) inequality, on the relative scale. These methods were repeated using a linear probability model to determine the SII, reflecting the absolute difference in multimorbidity prevalence between the highest and lowest incomes. An SII $>0$ is indicative of pro-rich inequality on the absolute scale. We also assessed trends in absolute inequality trends using pooled data. As a sensitivity test, we confirmed the statistical significance of these analyses adjusting for all measured determinants. We applied bootstrap sampling weights using balanced repeated replication $(n=500)$ to analyses. All data management was conducted using SAS Enterprise Guide version 6.1 (SAS Institute, Inc.) and all analyses were performed using Stata/MP version 13.1 (StataCorp).

\section{Results}

Of 134,395 Ontario respondents across 4 cycles of linked CCHS and administrative data, 122,002 (91\% of sample) were adults, and 113,627 (93\% of adults) had complete information across all variables for analysis. A higher proportion of adult respondents excluded (vs. included) from analyses were age $75+$ years, female, from the lowest income quintile and had prevalent multimorbidity. Table 1 describes the characteristics of respondents included in analyses for each CCHS cycle. The numbers outside and inside the brackets correspond to sample counts and population weighted proportions, respectively. Crude multimorbidity prevalence increased steadily with each cycle, from $26.4 \%$ in the 2005 survey to $33.5 \%$ in the 2011/12 survey. Crude prevalence estimates by household income quintile are shown in Fig. 1, and by determinant in the Additional file 1: Table S2. Of note, prevalence in the lowest vs. highest household income quintiles, respectively, were $34.7 \%$ vs. $20.8 \%$ in 2005 and $37.8 \%$ vs. $27.6 \%$ in $2011 / 12$.

The measured $\mathrm{C}_{\text {Erreygers }}$ are shown in the last row of Table 1: values ranged from -0.114 (CI: $-0.132,-0.096$ ) in 2005 to -0.085 (CI: $-0.108,-0.062$ ) in $2011 / 12$. The negative and statistically significant $\mathrm{C}_{\text {Erreygers }}$ indicates a greater concentration of multimorbidity among persons with low household income, although the smaller $C_{\text {Erreygers }}$ value in the 2011/12 survey suggests that inequality was less pronounced.

Table 2 summarizes the results of the $C_{\text {Erreygers }}$ decomposition of multimorbidity prevalence in the most recent (2011/12) and baseline (2005) surveys. Results from the $2007 / 08$ and $2009 / 10$ surveys are available in the Additional file 1: Table S3. Data include marginal effects from probit regressions, elasticities of each determinant $\left(\beta_{\mathrm{k}}{ }^{*} \mathrm{X}_{\mathrm{k}} / \mu\right)$, the $C_{\text {Erreygers }}$ of each determinant, and the relative contribution of each determinant to measured inequality. For example, for persons aged $75+$ in the 2011/12 CCHS cycle: the difference in conditional probability of multimorbidity (vs. 18-34 age group) was 77.3\%; this group represented $7.4 \%$ of the total population (from Table 1) but persons were concentrated in lower income levels $\left(C_{k}{ }^{*}\left(4^{*} \mu\right)=-0.061\right)$; and their contribution to measured income inequality was $55.6 \%$.

Individual determinants with the largest contribution to the observed pro-rich inequality in the 2011/12 CCHS cycle included low household income (57.2 and 18.8\% for quintiles 1 and 2, respectively), older age (55.6 and $29.9 \%$ for ages $75+$ and $65-74$ years, respectively), being divorced, separated, widowed or single (15.2\%), and physical inactivity (10.8\%). The relative contribution of each of these determinants to measured inequality increased from the decomposition analysis conducted using the 2005 CCHS survey data. For example, older ages (75+ years) were less concentrated among the low income (vs. 2005) but the elasticity (i.e., the \% change in multimorbidity associated with a \% change in the determinant) increased between cycles; for non-married 
Table 1 Characteristics of adult (age $\geq 18$ years) CCHS survey respondents linked to health administrative data: 2005 to $2011 / 12$

\begin{tabular}{|c|c|c|c|c|}
\hline Variable & CCHS 2005 & CCHS 2007-08 & CCHS 2009-10 & CCHS 2011/12 \\
\hline Total N: & 28,412 & 29,632 & 28,388 & 27,195 \\
\hline Prevalent Multimorbidity: & 9168 (26.4\%) & $11,156(30.3 \%)$ & $11,255(31.7 \%)$ & $11,390(33.5 \%)$ \\
\hline \multicolumn{5}{|l|}{ Age Group (years) } \\
\hline $18-34$ & $7324(29.6 \%)$ & $6563(29.0 \%)$ & $6471(28.8 \%)$ & $5988(28.8 \%)$ \\
\hline $35-49$ & 7417 (32.4\%) & $7311(30.3 \%)$ & $6323(29.2 \%)$ & $5557(27.2 \%)$ \\
\hline $50-64$ & $7046(22.2 \%)$ & $8152(24.4 \%)$ & $7790(25.3 \%)$ & 7706 (26.4\%) \\
\hline $65-74$ & 3645 (9.1\%) & $4132(9.4 \%)$ & 4177 (9.3\%) & $4452(10.1 \%)$ \\
\hline $75+$ & $2980(6.7 \%)$ & $3474(6.9 \%)$ & $3627(7.5 \%)$ & $3492(7.4 \%)$ \\
\hline \multicolumn{5}{|l|}{ Sex } \\
\hline Women & $15,322(51.2 \%)$ & $15,960(51.2 \%)$ & $15,565(51.3 \%)$ & $14,965(51.4 \%)$ \\
\hline Men & $13,090(48.8 \%)$ & $13,672(48.8 \%)$ & $12,823(48.7 \%)$ & $12,230(48.6 \%)$ \\
\hline \multicolumn{5}{|l|}{ Marital Status } \\
\hline Other (Divorced, separated, widowed or single) & $12,331(35.4 \%)$ & $12,537(35.9 \%)$ & $12,357(36.6 \%)$ & $12,150(37.8 \%)$ \\
\hline Married or common-law & $16,081(64.6 \%)$ & $17,095(64.1 \%)$ & $16,031(63.4 \%)$ & $15,045(62.2 \%)$ \\
\hline \multicolumn{5}{|l|}{ Immigrant } \\
\hline Born in Canada & $22,691(68.7 \%)$ & $23,322(67.0 \%)$ & $22,460(66.2 \%)$ & $21,739(67.6 \%)$ \\
\hline Not born in Canada & $5721(31.3 \%)$ & $6310(33.0 \%)$ & $5928(33.8 \%)$ & $5456(32.4 \%)$ \\
\hline \multicolumn{5}{|l|}{ Rurality } \\
\hline Urban $(\mathrm{RIO}<10)$ & $14,877(71.8 \%)$ & $15,734(72.2 \%)$ & $15,057(72.2 \%)$ & $14,342(72.6 \%)$ \\
\hline Suburban (RIO 10-39) & $8664(20.3 \%)$ & $9276(19.7 \%)$ & $8724(20.3 \%)$ & $8342(19.7 \%)$ \\
\hline Rural $(\mathrm{RIO}>40)$ & $4871(7.9 \%)$ & $4622(8.0 \%)$ & $4607(7.5 \%)$ & $4511(7.7 \%)$ \\
\hline \multicolumn{5}{|l|}{ Income (provincial quintile) } \\
\hline Q1 (Low) & $5617(18.6 \%)$ & $5190(18.3 \%)$ & $5079(18.7 \%)$ & $4460(17.9 \%)$ \\
\hline Q2 & $5719(19.8 \%)$ & $5793(19.2 \%)$ & $5431(19.0 \%)$ & $5140(19.1 \%)$ \\
\hline Q3 & $5305(19.8 \%)$ & $5700(19.8 \%)$ & $5749(20.7 \%)$ & 5825 (19.4\%) \\
\hline Q4 & $5776(20.7 \%)$ & $6385(21.3 \%)$ & $6005(20.3 \%)$ & $5648(21.6 \%)$ \\
\hline Q5 (High) & $5995(21.2 \%)$ & $6564(21.3 \%)$ & $6124(21.3 \%)$ & $6122(21.9 \%)$ \\
\hline \multicolumn{5}{|l|}{ Education (Individual) } \\
\hline No Post-Secondary Education & $10,488(32.4 \%)$ & $10,612(31.8 \%)$ & $9893(30.3 \%)$ & $9651(30.1 \%)$ \\
\hline At least Some Post-Secondary Education & $17,924(67.6 \%)$ & $19,020(68.2 \%)$ & $18,495(69.7 \%)$ & $17,544(69.9 \%)$ \\
\hline \multicolumn{5}{|l|}{ Physical Activity } \\
\hline Active & $7471(26.0 \%)$ & $7284(23.7 \%)$ & $7464(26.4 \%)$ & $7729(28.1 \%)$ \\
\hline Moderately Active & $7249(25.1 \%)$ & $7500(24.7 \%)$ & 7189 (24.4\%) & 7099 (25.8\%) \\
\hline Inactive & $13,692(49.0 \%)$ & $14,848(51.6 \%)$ & $13,735(49.2 \%)$ & $12,367(46.1 \%)$ \\
\hline \multicolumn{5}{|l|}{ Smoker } \\
\hline Heavy smoker (At least 1 pack per day) & $2233(6.4 \%)$ & 2079 (5.9\%) & $1809(5.1 \%)$ & $1619(4.7 \%)$ \\
\hline Light smoker (less than 1 pack per day) & $4723(16.4 \%)$ & $4540(15.9 \%)$ & $4116(14.8 \%)$ & 3987 (15.5\%) \\
\hline Former smoker & 7799 (24.2\%) & $8264(23.0 \%)$ & 7635 (21.7\%) & $7554(22.6 \%)$ \\
\hline Non-smoker & $13,657(53.1 \%)$ & $14,749(55.2 \%)$ & $14,828(58.4 \%)$ & $14,035(57.1 \%)$ \\
\hline \multicolumn{5}{|l|}{ Body-Mass Index (BMI) } \\
\hline Underweight (BMI < 18.5 kg/m2) & $679(2.8 \%)$ & $652(2.8 \%)$ & $603(2.6 \%)$ & $625(2.6 \%)$ \\
\hline Normal weight (BMl 18.5-24.9kg/m2) & $12,558(47.4 \%)$ & $12,405(45.3 \%)$ & $11,888(45.4 \%)$ & $11,250(44.7 \%)$ \\
\hline Overweight (BMI 25-29.9 kg/m2) & 9915 (34.0\%) & $10,530(34.8 \%)$ & 9955 (34.0\%) & $9486(34.2 \%)$ \\
\hline Obese (BMl > 30 kg/m2) & $5260(15.8 \%)$ & $6045(17.2 \%)$ & $5942(18.0 \%)$ & $5834(18.5 \%)$ \\
\hline
\end{tabular}


Table 1 Characteristics of adult (age $\geq 18$ years) CCHS survey respondents linked to health administrative data: 2005 to $2011 / 12$ (Continued)

\begin{tabular}{|c|c|c|c|c|}
\hline Variable & CCHS 2005 & CCHS 2007-08 & CCHS 2009-10 & CCHS 2011/12 \\
\hline \multicolumn{5}{|l|}{ Local Health Integration Network (LHIN) } \\
\hline Erie St. Clair & $2102(5.0 \%)$ & $1985(5.0 \%)$ & $2030(4.9 \%)$ & $1933(4.8 \%)$ \\
\hline South West & $3706(7.4 \%)$ & $3631(7.3 \%)$ & $3588(7.0 \%)$ & $3282(7.4 \%)$ \\
\hline Waterloo-Wellington & 1709 (5.4\%) & $1715(5.4 \%)$ & $1729(5.6 \%)$ & $1570(5.7 \%)$ \\
\hline Hamilton Niagara Haldimand Brant (HNHB) & $3642(11.0 \%)$ & $3404(10.5 \%)$ & $3380(11.0 \%)$ & $3284(10.7 \%)$ \\
\hline Central West & $862(5.9 \%)$ & $1296(7.3 \%)$ & $1210(5.8 \%)$ & $1143(5.8 \%)$ \\
\hline Mississauga Halton & $1378(8.9 \%)$ & $1437(8.6 \%)$ & 1369 (8.7\%) & $1410(9.4 \%)$ \\
\hline Toronto Central & $1058(9.7 \%)$ & $1377(9.0 \%)$ & 1177 (9.2\%) & $1127(9.7 \%)$ \\
\hline Central & $1632(11.8 \%)$ & $1779(12.6 \%)$ & $1657(13.0 \%)$ & 1487 (13.0\%) \\
\hline Central East & $2759(12.2 \%)$ & $2904(11.7 \%)$ & $2537(12.0 \%)$ & $2471(11.3 \%)$ \\
\hline South East & $1894(3.9 \%)$ & $1846(3.8 \%)$ & $1842(3.8 \%)$ & 1759 (3.7\%) \\
\hline Champlain & $2853(9.4 \%)$ & $2814(9.2 \%)$ & $2753(9.4 \%)$ & $2784(9.4 \%)$ \\
\hline North Simcoe Muskoka & 1059 (3.2\%) & $1448(3.5 \%)$ & $1310(3.5 \%)$ & $1287(3.3 \%)$ \\
\hline North East & $2727(4.5 \%)$ & $2677(4.5 \%)$ & 2455 (4.3\%) & $2365(4.2 \%)$ \\
\hline North West & $1031(1.8 \%)$ & $1319(1.6 \%)$ & $1351(1.6 \%)$ & $1293(1.6 \%)$ \\
\hline \multicolumn{5}{|l|}{ Primary Care Model Affiliation } \\
\hline Family Health Group (FHG) & $7068(23.6 \%)$ & $11,616(42.7 \%)$ & 7765 (35.0\%) & $5648(27.7 \%)$ \\
\hline Family Health Network (FHN) & $2160(5.3 \%)$ & $4244(9.2 \%)$ & 1957 (4.0\%) & $1279(2.4 \%)$ \\
\hline Family Health Organization (FHO) & $1026(3.3 \%)$ & $3414(10.4 \%)$ & $10,566(30.6 \%)$ & $13,292(42.1 \%)$ \\
\hline Not Enrolled & $18,035(67.3 \%)$ & $8510(33.1 \%)$ & $6114(24.9 \%)$ & $5253(23.2 \%)$ \\
\hline Other Model & $123(0.4 \%)$ & $1848(4.6 \%)$ & $1986(5.5 \%)$ & $1723(4.6 \%)$ \\
\hline Concentration Index ${ }^{a}$ & $-0.114^{*}$ & $-0.114^{*}$ & $-0.111^{*}$ & $-0.085^{*}$ \\
\hline (95\% Confidence Interval) & $(-0.132,-0.096)$ & $(-0.133,-0.094)$ & $(-0.132,-0.089)$ & $(-0.108,-0.062)$ \\
\hline
\end{tabular}

${ }^{*} p<0.05$

${ }^{a}$ Concentration Index, Erreygers-corrected

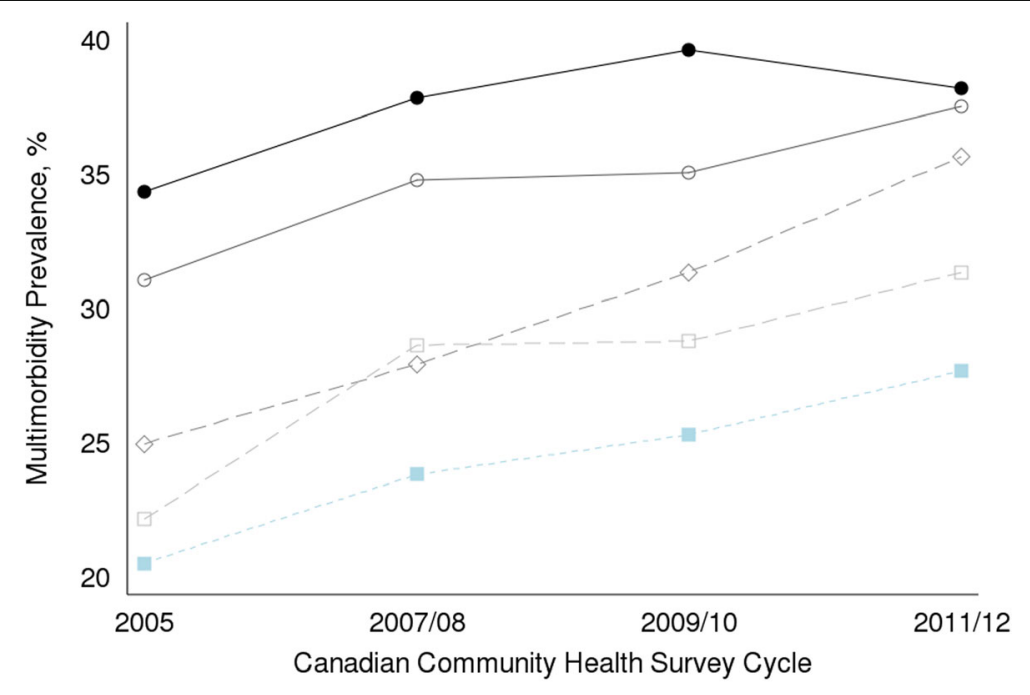

$\bullet$ Q1(Low) $\ominus$ Q2 $\Leftrightarrow$ Q3 $\quad$ Q4 $\quad$ Q5 (High)

Fig. 1 Longitudinal trends in multimorbidity by household income over four cycles of the CCHS 
Table 2 Decomposition of the Erreygers-corrected Concentration Index of multimorbidity prevalence among adults in Ontario: 2005 vs. $2011 / 12$

\begin{tabular}{|c|c|c|c|c|c|c|c|c|}
\hline \multirow[b]{2}{*}{ Variable } & \multicolumn{4}{|c|}{ CCHS 2005, $N=28,412$} & \multicolumn{4}{|c|}{ CCHS 2011-12, N=27,195 } \\
\hline & Marg. Effects & Elas. & $C_{k}$ & $\%$ Contr. & Marg. Effects & Elas. & $C_{k}$ & $\%$ Contr. \\
\hline \multicolumn{9}{|l|}{ Age Group (years) [REF = 18-34y] } \\
\hline $35-49$ & $0.15^{*}$ & 0.183 & 0.097 & -12.7 & $0.161^{*}$ & 0.131 & 0.065 & -12.2 \\
\hline $50-64$ & $0.339^{*}$ & 0.286 & 0.095 & -28.2 & $0.366^{*}$ & 0.289 & 0.120 & -51.6 \\
\hline $65-74$ & $0.489^{*}$ & 0.168 & -0.072 & 30.9 & $0.585^{*}$ & 0.177 & -0.044 & 29.9 \\
\hline v75+ & $0.637^{*}$ & 0.162 & -0.086 & 48.2 & $0.773^{*}$ & 0.17 & -0.061 & 55.6 \\
\hline \multicolumn{9}{|l|}{ Sex $[R E F=$ Women $]$} \\
\hline Men & $-0.066^{*}$ & -0.121 & 0.123 & 7.1 & $-0.056^{*}$ & -0.081 & 0.113 & 7.4 \\
\hline \multicolumn{9}{|l|}{ Marital Status [REF = Married $]$} \\
\hline Other & $0.039^{*}$ & 0.052 & -0.194 & 6.6 & $0.05^{*}$ & 0.057 & -0.258 & 15.2 \\
\hline \multicolumn{9}{|l|}{ Immigrant [REF=Born in Canada] } \\
\hline Not born in Canada & -0.003 & -0.003 & -0.225 & -0.6 & $-0.03^{*}$ & -0.029 & -0.225 & -7.8 \\
\hline \multicolumn{9}{|l|}{ Rurality [REF=Urban] } \\
\hline Suburban & $-0.019^{*}$ & -0.015 & 0.042 & 0.7 & -0.019 & -0.011 & 0.066 & 1.5 \\
\hline Rural & -0.016 & -0.005 & 0.018 & 0.3 & $-0.034^{*}$ & -0.008 & 0.028 & 1.1 \\
\hline \multicolumn{9}{|l|}{ Income (quintile) [REF = Q5 (high)] } \\
\hline Q1 (Low) & $0.06^{*}$ & 0.042 & -0.605 & 31.9 & $0.083^{*}$ & 0.044 & -0.589 & 57.2 \\
\hline Q2 & $0.034^{*}$ & 0.025 & -0.341 & 10.1 & $0.047^{*}$ & 0.027 & -0.344 & 18.8 \\
\hline Q3 & 0.011 & 0.008 & -0.027 & 0.3 & $0.06^{*}$ & 0.034 & -0.050 & 3.5 \\
\hline Q4 & 0.013 & 0.01 & 0.306 & -3.4 & 0.03 & 0.019 & 0.299 & -10.5 \\
\hline \multicolumn{9}{|l|}{ Education [REF=Some post-sec.] } \\
\hline No Post-Secondary Education & 0.001 & 0.001 & -0.252 & 0.2 & 0.011 & 0.01 & -0.242 & 3.2 \\
\hline \multicolumn{9}{|l|}{ Physical Activity [REF = Active] } \\
\hline Moderately Active & $0.028^{*}$ & 0.026 & 0.064 & -1.6 & 0 & 0 & 0.067 & 0 \\
\hline Inactive & $0.027^{*}$ & 0.051 & -0.159 & 3.8 & $0.048^{*}$ & 0.066 & -0.193 & 10.8 \\
\hline \multicolumn{9}{|l|}{ Smoker [REF=Non-smoker] } \\
\hline Heavy smoker & $0.039^{*}$ & 0.009 & -0.016 & 0.5 & $0.05^{*}$ & 0.007 & -0.030 & 1.8 \\
\hline Light smoker & 0.021 & 0.013 & -0.031 & 0.6 & 0.018 & 0.008 & -0.068 & 1.4 \\
\hline Former smoker & $0.038^{*}$ & 0.035 & 0.045 & -1.5 & $0.055^{*}$ & 0.037 & 0.061 & -4 \\
\hline \multicolumn{9}{|l|}{ BMI [REF=Normal weight] } \\
\hline Underweight & -0.005 & -0.001 & -0.021 & -0.1 & -0.044 & -0.003 & -0.024 & -1.3 \\
\hline Overweight & $0.049^{*}$ & 0.063 & 0.043 & -1.8 & $0.072^{*}$ & 0.073 & 0.041 & -3.4 \\
\hline Obese & $0.154^{*}$ & 0.092 & -0.022 & 2.9 & $0.171^{*}$ & 0.095 & -0.002 & 0.4 \\
\hline \multicolumn{9}{|l|}{ LHIN [REF = Erie St. Clair $]$} \\
\hline South West & $-0.035^{*}$ & -0.01 & 0.021 & 0.6 & $-0.061^{*}$ & -0.013 & 0.008 & 0.6 \\
\hline Waterloo-Wellington & $-0.102^{*}$ & -0.021 & 0.022 & 2.0 & $-0.074^{*}$ & -0.013 & 0.014 & 1.2 \\
\hline $\mathrm{HNHB}$ & -0.018 & -0.007 & -0.008 & -0.1 & $-0.077^{*}$ & -0.025 & -0.002 & -0.2 \\
\hline Central West & -0.028 & -0.006 & -0.033 & -0.8 & $-0.068^{*}$ & -0.012 & -0.031 & -2.4 \\
\hline Mississauga Halton & -0.04 & -0.014 & 0.008 & 0.3 & -0.01 & -0.003 & 0.003 & 0 \\
\hline Toronto Central & -0.013 & -0.005 & -0.018 & -0.2 & $-0.097^{*}$ & -0.028 & -0.003 & -0.3 \\
\hline Central & $-0.042^{*}$ & -0.019 & -0.021 & -0.8 & $-0.089^{*}$ & -0.034 & -0.030 & -3.1 \\
\hline Central East & -0.013 & -0.006 & -0.018 & -0.2 & -0.047 & -0.016 & -0.027 & -1.5 \\
\hline
\end{tabular}


Table 2 Decomposition of the Erreygers-corrected Concentration Index of multimorbidity prevalence among adults in Ontario: 2005 vs. 2011/12 (Continued)

\begin{tabular}{|c|c|c|c|c|c|c|c|c|}
\hline \multirow[b]{2}{*}{ Variable } & \multicolumn{4}{|c|}{ CCHS 2005, N=28,412 } & \multicolumn{4}{|c|}{ CCHS $2011-12, N=27,195$} \\
\hline & Marg. Effects & Elas. & $C_{k}$ & $\%$ Contr. & Marg. Effects & Elas. & $C_{k}$ & $\%$ Contr. \\
\hline South East & $-0.042^{*}$ & -0.006 & 0.006 & 0.2 & -0.051 & -0.006 & 0.007 & 0.4 \\
\hline Champlain & $-0.037^{*}$ & -0.013 & 0.024 & 0.8 & -0.042 & -0.012 & 0.039 & 1.9 \\
\hline North Simcoe Muskoka & $-0.051^{*}$ & -0.006 & 0.011 & 0.5 & -0.056 & -0.006 & 0.016 & 1.1 \\
\hline North East & $-0.048^{*}$ & -0.008 & -0.008 & -0.3 & -0.046 & -0.006 & 0.008 & 0.4 \\
\hline North West & $-0.075^{*}$ & -0.005 & 0.001 & 0.1 & $-0.12^{*}$ & -0.006 & 0.006 & 0.8 \\
\hline \multicolumn{9}{|c|}{ Primary Care Model [REF=None] } \\
\hline Family Health Group & $0.058^{*}$ & 0.052 & 0.029 & -1.5 & $0.154^{*}$ & 0.128 & -0.042 & 7.5 \\
\hline Family Health Network & 0.015 & 0.003 & 0.019 & -0.3 & $0.104^{*}$ & 0.008 & 0.011 & -1.3 \\
\hline Family Health Org. & $-0.052^{*}$ & -0.007 & -0.012 & -0.6 & $0.105^{*}$ & 0.132 & 0.093 & -11.5 \\
\hline Other Model & 0.058 & 0.001 & 0.000 & 0.0 & $0.121^{*}$ & 0.017 & 0.015 & -2.1 \\
\hline \multicolumn{3}{|l|}{ Sum (of $C_{\text {Erreygers }}$ ) } & -0.107 & 93.8 & & & -0.093 & 108.6 \\
\hline \multicolumn{3}{|l|}{ Residual (unexplained) } & \multicolumn{2}{|l|}{-0.007} & & & \multicolumn{2}{|l|}{0.007} \\
\hline \multicolumn{3}{|c|}{ Erreygers-corrected Concentration Index } & \multicolumn{2}{|l|}{-0.114} & & & \multicolumn{2}{|l|}{-0.085} \\
\hline
\end{tabular}

*indicates statistically significant $(p<0.05)$ marginal effect derived from multivariable probit regression model Elas. $=$ Elasticity $\left(\beta_{\mathrm{k}}{ }^{*} \overline{\mathrm{x}}_{\mathrm{k}} / \mu\right) ; C_{\mathrm{k}}=$ Erreygers-corrected Concentration Index of determinant $\mathrm{k}$

persons, the elasticity increased, as did the concentration of non-married individuals among lower income groups. In contrast, variables that contributed negatively to inequality in the latter survey included being middle-aged $(-51.6 \%$ and $-12.2 \%$ for $50-64$ and $35-49$ years, respectively), having higher household income $(-10.5 \%$ for quintile 4), enrolment in a FHO $(-11.5 \%)$ and being born outside of Canada $(-7.8 \%)$. Of note, the growth in FHO enrolment between surveys (from 3.3 to 42.1\%) corresponded with a greater concentration among higher household income $\left(C_{\text {Erreygers }}\right.$ from -0.012 to 0.093$)$; the opposite pattern was found for enrolment in a FHG (increased in enrolment from 23.6 to $27.7 \%$ and shift in $\mathrm{C}_{\text {Erreygers }}$ from 0.029 to -0.042 ).

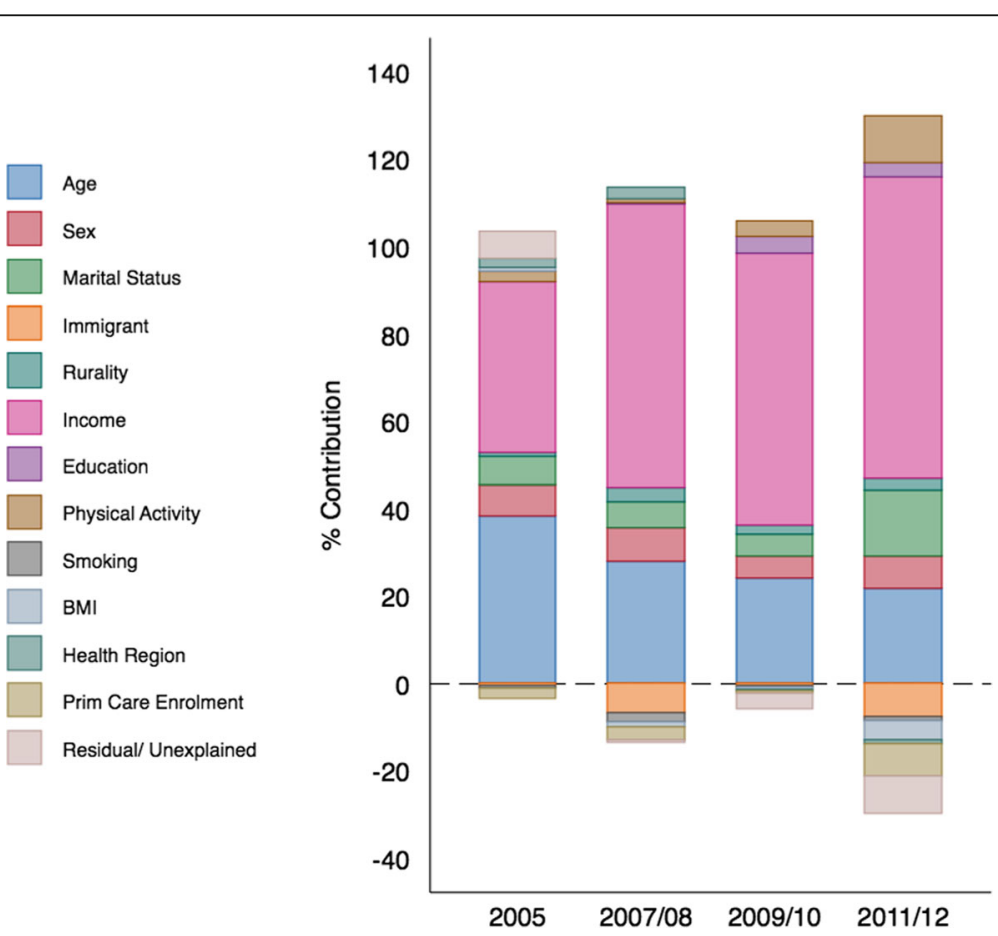

Fig. 2 Summary of the relative contribution of key determinants to income inequality in multimorbidity in Ontario, 2005 to 2011/12 
Aggregating these percentages by determinant (Fig. 2), income explained $69.0 \%$ of the measured inequality in 2011/12 (an increase of $+30.2 \%$ from 2005 CCHS survey), followed by age at $21 \%(-16.5 \%)$, marital status at $15.2 \%(+8.6 \%)$, and physical activity at $10.9 \%(+8.6 \%)$. In contrast, determinants that contributed the largest to decreasing inequality were immigration at $-7.8(-7.2 \%)$ and primary care enrolment at $-7.4(-5.0 \%)$. In 2011/ 12 , the modeled determinants explained -0.093 of the total inequality $(-0.085)$, whereas in 2005 the determinants explained -0.107 of the total inequality $(-0.114)$.

Table 3 shows the age-sex adjusted relative (RII) and absolute (SII) inequality measures for each survey, as well as the longitudinal trend analysis. The prevalence rate ratio (RII) in 2011/12 comparing high vs. low income quintile was 1.32 (CI: 1.19, 1.46) in 2011/12. The corresponding prevalence rate difference (SII) was $9.1 \%$ (CI: 5.7, 12.4\%). In pooled regression analyses, longitudinal trends in inequality were not statistically significant over the study period on either the absolute or relative scale, suggesting no change in measured income inequality ( $\mathrm{p}$ for interaction $=0.405$ for RII and 0.443 for SII).

These findings were robust to the defined sensitivity analyses (Additional file 1: Table S3).

\section{Discussion}

This study confirms a moderate, statistically significant pro-rich household income gradient in multimorbidity prevalence in Ontario, Canada. This gap was observed on both relative and absolute scales, and has persisted from 2005 to 2011/12. Our results are consistent with measured wealth and socioeconomic inequalities in chronic conditions among adults observed in low and middle-income countries $[19,56,58]$. These studies considered only a small number of conditions in defining

Table 3 Trends in income inequalities in multimorbidity prevalence among adults in Ontario, 2005 to 2011/12

\begin{tabular}{|c|c|c|c|c|c|}
\hline CCHS Cycle: & 2005 & $2007 / 08$ & $2009 / 10$ & $2011 / 12$ & Trend \\
\hline \multicolumn{6}{|c|}{ Relative Index of Inequality } \\
\hline RII (ridit score) & $1.376^{*}(1.233,1.535)$ & $1.405 *(1.280,1.543)$ & $1.427^{*}(1.290,1.579)$ & $1.321^{*}(1.191,1.466)$ & $1.457^{*}(1.288,1.649)$ \\
\hline \multicolumn{6}{|l|}{ Age } \\
\hline $35-49$ & $2.402 *(2.093,2.756)$ & $2.323^{*}(2.007,2.689)$ & $2.297^{*}(2.011,2.624)$ & $2.039^{*}(1.742,2.387)$ & $2.240^{*}(2.081,2.412)$ \\
\hline $50-64$ & $5.531 *(4.865,6.287)$ & $5.031^{*}(4.408,5.741)$ & $4.649 *(4.151,5.207)$ & $4.116^{*}(3.585,4.726)$ & $4.737^{*}(4.437,5.057)$ \\
\hline $65-74$ & $8.098 *(7.164,9.155)$ & $7.757^{*}(6.825,8.816)$ & $7.406^{*}(6.620,8.287)$ & $6.293^{*}(5.553,7.131)$ & $7.269^{*}(6.843,7.721)$ \\
\hline $75+$ & $10.287^{*}(9.152,11.564)$ & $9.017^{*}(7.936,10.245)$ & $8.761^{*}(7.859,9.767)$ & $7.667^{*}(6.742,8.719)$ & $8.762^{*}(8.251,9.304)$ \\
\hline \multicolumn{6}{|l|}{ Sex } \\
\hline Male & $0.832 *(0.791,0.876)$ & $0.853^{*}(0.808,0.901)$ & $0.911^{*}(0.862,0.963)$ & $0.884^{*}(0.836,0.935)$ & $0.873^{*}(0.849,0.898)$ \\
\hline CCHS Cycle & & & & & $1.067^{*}(1.036,1.099)$ \\
\hline RII*Cycle & & & & & $0.981(0.937,1.027)$ \\
\hline Constant & $0.065(0.052,0.074)^{*}$ & $0.076(0.067,0.087)^{*}$ & $0.079(0.070,0.089)^{*}$ & $0.098(0.085,0.113)$ & $0.067(0.061,0.074)^{*}$ \\
\hline \multicolumn{6}{|c|}{ Slope Index of Inequality } \\
\hline SII (ridit score) & $0.082 *(0.054,0.109)$ & $0.099 *(0.072,0.127)$ & $0.110^{*}(0.079,0.141)$ & $0.091^{*}(0.057,0.124)$ & $0.082^{*}(0.047,0.116)$ \\
\hline \multicolumn{6}{|l|}{ Age } \\
\hline $35-49$ & $0.100 *(0.085,0.115)$ & $0.114^{*}(0.095,0.132)$ & $0.120^{*}(0.101,0.139)$ & $0.113^{*}(0.089,0.137)$ & $0.112^{*}(0.102,0.122)$ \\
\hline $50-64$ & $0.316^{*}(0.294,0.338)$ & $0.338^{*}(0.316,0.360)$ & $0.330 *(0.307,0.352)$ & $0.332 *(0.305,0.358)$ & $0.329 *(0.317,0.341)$ \\
\hline $65-74$ & $0.509^{*}(0.483,0.535)$ & $0.581^{*}(0.554,0.608)$ & $0.592 *(0.566,0.618)$ & $0.571^{*}(0.546,0.596)$ & $0.564^{*}(0.551,0.577)$ \\
\hline $75+$ & $0.681^{*}(0.657,0.706)$ & $0.703 *(0.677,0.729)$ & $0.726^{*}(0.703,0.749)$ & $0.730 *(0.703,0.756)$ & $0.711^{*}(0.699,0.723)$ \\
\hline \multicolumn{6}{|l|}{ Sex } \\
\hline Male & $-0.048 *(-0.062,-0.035)$ & $-0.048^{*}(-0.064,-0.032)$ & $-0.030 *(-0.048,-0.013)$ & $-0.042^{*}(-0.060,-0.023)$ & $-0.042 *(-0.050,-0.034)$ \\
\hline CCHS Cycle & & & & & $0.013^{*}(0.005,0.021)$ \\
\hline SII*Cycle & & & & & $0.005(-0.008,0.019)$ \\
\hline Constant & $0.053^{*}(0.035,0.071)$ & $0.056^{*}(0.037,0.075)$ & $0.048^{*}(0.028,0.069)$ & $0.079 *(0.054,0.104)$ & $0.026^{*}(0.005,0.046)$ \\
\hline Observations, N: & 28,412 & 29,632 & 28,388 & 27,195 & 113,627 \\
\hline
\end{tabular}

${ }^{*} p<0.05$

Data are regression coefficients from a Poisson regression (relative index of inequality, RII) or a linear probability model (slope index of inequality, SII) Estimates and corresponding standard errors were estimated with bootstrap sampling weights using balanced repeated replication

For RII*Cycle, a statistically significant interaction with value $>1(<1)$ is indicative of increasing (decreasing) inequality on the relative scale over time; for the SII*Cycle, a positive (negative) and statistically significant interaction is indicative of increasing (decreasing) inequality on the absolute scale over time 
chronic disease burden. Our findings are also consistent with a study of multimorbidity (among 52 conditions) in deprived areas in the Basque Country of Spain [59]. Although socioeconomic differences in common chronic conditions among adult populations have been previously reported [60,61], few studies have considered an aggregated count of multiple high impact chronic conditions or measured inequality with robust methods that account for the entire socio-economic distribution.

These findings highlight the unequal distribution of multimorbidity across socio-economic ranks. An important contribution of the current study to existing literature is the measurement of the relative contributions of multimorbidity's determinants to measured income inequalities from a high-income setting. The decomposition method is useful for policy makers who seek to reduce health inequalities by targeting determinants that contribute the most to observed inequalities. Recently, Kunna et al. [19] decomposed wealth-related inequalities in multimorbidity prevalence (defined as 2 or more of 7 chronic conditions) among adults aged 50 years and older in China (middle-income setting) and in Ghana (low-income setting). Considering a similar set of measured determinants, the authors found that largest contributors to inequality were wealth, age, and education in China and BMI, wealth and rurality in Ghana. We found that the largest contributor to household income inequality in multimorbidity prevalence was income itself. This finding has been observed for similar decomposition studies of income-related inequalities in general health status from North America [62] and Europe [63]. Our data also show that the proportion of inequality in multimorbidity due to income increased over time. The growth in the rich-poor income gap in Canada, like may comparable OECD nations, has been well documented [37].

The strong negative association between increasing age and multimorbidity prevalence we observed is well established in existing literature [16]. The positive contribution of age to measured inequality in our study decreased steadily between cycles, mainly due to changes among the 50-64 year age group, who became more concentrated among higher household incomes. Among other socio-demographic variables, the contribution of marital status to measured inequality increased. Similar to age, where the change to the elasticity was minor, the increase in contribution was driven by a greater concentration of divorced, separated, widowed or single individuals in lower household income levels.

Physical activity was the strongest lifestyle factor contributing to observed inequalities in multimorbidity. We found that prevalence of multimorbidity increased over the study period (from 29.4 to $39.5 \%$ ) among inactive persons in Ontario, and that inactivity was increasingly concentrated among lower incomes. Socioeconomic gradients in inactivity have been documented in other high-income jurisdictions [64], although less is known regarding changes across the socioeconomic distribution over time. Body-mass index had only a small negative contribution to inequality overall, and obesity in particular was relatively equally distributed across income. This contrasts findings by Hajizahen et al. [65], who report a small but significant concentration of obesity among the poor in Canada. Their data, however, exclude older adults (aged 65+ years). Our findings show that heavy and former smoking were unequally distributed across income but had small (although statistically significant) regression effects and small corresponding elasticities, also resulting in a minor contribution to inequality. The variability in the contribution of lifestyle determinants to inequalities in multimorbidity from previous work from low and middle-income jurisdictions [19] suggest that interventions meant to improve equality need to be targeted to local contexts. Other unhealthy lifestyle factors that are potentially amenable to public health intervention, such as healthy diet and alcohol consumption, were not included in our multivariable decomposition analyses since their relationships with multimorbidity prevalence are less certain [40].

The contribution of health system variables to overall inequality, including health region of residence and enrolment in a primary care model, were small. All participants of the study had universal access to medically necessary care; however, enrolment into a primary care model is voluntary. The negative contributions for this determinant in particular suggest that holding all else constant, persons enrolled in these models (particularly FHNs) are less likely to be multimorbid and of higher household income, which has been previously documented [44, 45]. Lastly, across all decomposition analyses, being born outside of Canada was negatively associated with multimorbidity prevalence (to varying effect sizes), and negatively contributed to multimorbidity inequality. A healthy immigrant effect for chronic disease incidence has been previously documented in Canada, although with diminishing associations as immigrants' time in Canada lengthened [66, 67]. The effect of time since immigrating on socioeconomic inequalities in multimorbidity (and other health outcomes) is an avenue for future research.

The results from the decomposition were robust to multiple sensitivity analyses. Other strengths of the research include the use of a large sample size, survey weights that are reflective of Ontario's population, and high survey response rates. Our results are likely to be generalizable to other high-income jurisdictions with comparable universal health care systems. Validated algorithms were also used to ascertain disease status; linked survey and administrative data enabled this 
inclusion. There are limitations of this research that are worth noting, however. Variables were defined using routinely-collected health administrative and survey data which are subject to known limitations [22, 23]. For health administrative data in particular, ascertainment of chronic disease is dependent having a place of residence and on health system contact (physician visits or hospital admissions), which are less likely among vulnerable, low-SES individuals. This would underreport multimorbidity prevalence and the overall measured inequality. Each survey is cross-sectional by design, and therefore, causality cannot be inferred from the data or by the decompositions. Inequality trends were assessed over a 7 -year period from biennial cross-sectional surveys; the inclusion of additional surveys could be an avenue for future research, once linked to administrative data. Importantly, interpretation of decomposition results rely on a correct model specification; Pseudo R-squared values from the multivariable probit models were 0.22 (2005 CCHS) and 0.23 (2011/12 CCHS) suggesting the variables included in the regression explain only a portion of the variance observed in the outcome. Lastly, we were only able to include program enrolment as a marker of primary care access. However, a high-performing primary care sector can support equitable health outcomes by promoting seamless access to services across the care continuum, including preventive care and ongoing chronic disease management [68-70]. As such, other access-related determinants not measurable with our data may contribute to income inequalities in multimorbidity prevalence. Additional research that includes primary care capacities could help inform policymakers, and play a pivotal role for reducing overall disparities.

\section{Conclusions}

This study confirms a pro-rich income gap in multimorbidity prevalence that has persisted over time. This gap is driven primarily by income, increasing age, marital status (not being married or common-law) and physical inactivity. Additional research to uncover the root causes of co-existing chronic conditions and to develop and evaluate the effectiveness of prevention strategies are warranted to Acknowledgements.

We thank IMS Brogan Inc. for use of their Drug Information Database.

\section{Additional file}

Additional file 1: Table S1. Multimorbidity prevalence (standard deviation) by determinant. Table S2. Decomposition results from 2007/08 and 2009/10 CCHS surveys. Table S3. Sensitivity Results. (DOCX $49 \mathrm{~kb}$ )

\section{Abbreviations}

BMl: Body mass index; C: Concentration index; CAPE: Client Agency Program Enrolment database; CCHS: Canadian Community Health Survey;
$C_{\text {Erreygers: }}$ Erreygers-corrected concentration index; DAD: Discharge Abstract Database; ICES: Institute for Clinical Evaluative Sciences; LHIN: Local Health Integration Network; ODB: Ontario Drug Benefit database; OECD: Organisation for Economic Co-operation and Development; OHIP: Ontario Health Insurance Program claims database; Rll: Relative index of inequality; SII: Slope index of inequality

\section{Acknowledgements}

We thank IMS brogan Inc. for use of their drug information Database.

\section{Funding}

This study was funded by the Health System Performance Research Network (HSPRN) and supported by the Institute for Clinical Evaluative Sciences (ICES), which is funded by an annual grant from the Ontario Ministry of Health and Long-Term Care (MOHLTC). The opinions, results and conclusions reported in this paper are those of the authors and are independent from the funding sources. No endorsement by ICES or the MOHLTC is intended or should be inferred. Parts of this material are also based on data and information compiled and provided by the Canadian Institute for Health Information (ClHI). However, the analyses, conclusions, opinions and statements expressed herein are those of the authors, and not necessarily those of $\mathrm{ClHI}$.

\section{Availability of data and materials}

The data from this study is held securely in coded form at the Institute for Clinical Evaluative Sciences (ICES). While data sharing agreements prohibit ICES from making the data set publicly available, access may be granted to those who meet pre-specified criteria for confidential access, available at www.ices.on.ca/DAS (contact: das@ices.on.ca).

\section{Authors' contributions}

All authors contributed to the conception and design of the research study. LM generated the analytical cohort, performed all statistical analyses, and prepared the original draft of the manuscript. All co-authors contributed intellectual content to subsequent revisions. All co-authors have approved the final draft for submission and take public responsibility for it. All co-authors have read, and confirm that they meet ICMJE criteria for authorship.

Ethics approval and consent to participate

We used survey data and linked health information that is routinely collected in Ontario that did not require informed consent from study participants. The use of this data was authorized under section 45 of Ontario's Personal Health Information Protection Act, which does not require review by a Research Ethics Board.

Consent for publication

Not applicable.

\section{Competing interests}

The authors declare that they have no competing interests.

\section{Publisher's Note}

Springer Nature remains neutral with regard to jurisdictional claims in published maps and institutional affiliations.

\section{Author details}

${ }^{1}$ Institute for Clinical Evaluative Sciences (ICES), G1 062075 Bayview Ave, Toronto, ON M4N 3M5, Canada. ${ }^{2}$ Health System Performance Research Network (HSPRN), 155 College St 4th Floor, Toronto, ON M5T 3M6, Canada. ${ }^{3}$ School of Epidemiology and Public Health, University of Ottawa, 600 Peter Morand Crescent, Ottawa, ON K1G Z53, Canada. ${ }^{4}$ Institute of Health Policy, Management, and Evaluation (IHPME), University of Toronto, 155 College St 4th Floor, Toronto, ON M5T 3M6, Canada. ${ }^{5}$ Institute for Better Health, Trillium Health Partners, 100 Queensway West, Mississauga, ON L5B 1B8, Canada. 
Received: 4 December 2017 Accepted: 11 June 2018 Published online: 26 June 2018

\section{References}

1. Koné Pefoyo AJ, Bronskill SE, Gruneir A, Calzavara A, Thavorn K, Petrosyan Y, et al. The increasing burden and complexity of multimorbidity. BMC Public Health. 2015:15:415. BioMed Central Ltd

2. Rijken M, van Kerkhof M, Dekker J, Schellevis FG. Comorbidity of chronic diseases: effects of disease pairs on physical and mental functioning. Qual Life Res. 2005;14:45-55

3. Tinetti ME, McAvay GJ, Chang SS, Newman AB, Fitzpatrick AL, Fried TR, et al. Contribution of multiple chronic conditions to universal health outcomes. J Am Geriatr Soc. 2011:59:1686-91.

4. Nunes BP, Flores TR, Mielke Gl, Thumé E, Facchini LA. Multimorbidity and mortality in older adults: a systematic review and meta-analysis. Arch Gerontol Geriatr. 2016;67:130-8. Elsevier Ireland Ltd

5. Bähler C, Huber CA, Brüngger B, Reich O. Multimorbidity, health care utilization and costs in an elderly community-dwelling population: a claims data based observational study. BMC Health Serv Res. 2015;15:23. BioMed Central Ltd

6. Glynn LG, Valderas JM, Healy P, Burke E, Newell J, Gillespie P, et al. The prevalence of multimorbidity in primary care and its effect on health care utilization and cost. Fam Pract. 2011;28:516-23.

7. Wolff JL, Starfield B, Anderson G. Prevalence, expenditures, and complications of multiple chronic conditions in the elderly. Arch Intern Med. 2002:162:2269-76.

8. Smith SM, Soubhi H, Fortin M, Hudon C, O'Dowd T. Managing patients with multimorbidity: systematic review of interventions in primary care and community settings. BMJ. 2012;345:e5205.

9. Kuo RN, Lai M-S. The influence of socio-economic status and multimorbidity patterns on healthcare costs: a six-year follow-up under a universal healthcare system. Int J Equity Health. 2013;12:69.

10. van Oostrom SH, Picavet HSJ, de Bruin SR, Stirbu I, Korevaar JC, Schellevis FG, et al. Multimorbidity of chronic diseases and health care utilization in general practice. BMC Fam Pract. 2014;15:61. BioMed Central

11. Zulman DM, Pal Chee C, Wagner TH, Yoon J, Cohen DM, Holmes TH, et al. Multimorbidity and healthcare utilisation among high-cost patients in the US veterans affairs health care system. BMJ Open. 2015;5:e007771. British Medical Journal Publishing Group

12. Gruneir A, Bronskill SE, Maxwell CJ, Bai YQ, Kone AJ, Thavorn K, et al. The association between multimorbidity and hospitalization is modified by individual demographics and physician continuity of care: a retrospective cohort study. BMC Health Serv Res. 2016;16:1-9. 2nd ed. BMC Health Serv Res

13. Roberts KC, Rao DP, Bennett TL, Loukine L, Jayaraman GC. Prevalence and patterns of chronic disease multimorbidity and associated determinants in Canada. Health Promot Chronic Dis Prev Can. 2015;35:87-94.

14. Lane NE, Maxwell CJ, Gruneir A, Bronskill SE, Wodchis WP. Absence of a socioeconomic gradient in older Adults' survival with multiple chronic conditions. EBioMedicine. 2015;2:2094-100.

15. Salisbury C, Johnson L, Purdy S, Valderas JM, Montgomery AA Epidemiology and impact of multimorbidity in primary care: a retrospective cohort study. Br J Gen Pract. 2011;61:12-21.

16. Violán C, Foguet-Boreu Q, Roso-Llorach A, Rodriguez-Blanco T, Pons-Vigués M, Pujol-Ribera E, et al. Burden of multimorbidity, socioeconomic status and use of health services across stages of life in urban areas: a cross-sectional study. BMC Public Health. 2014;14:530-13. Available from: http://www. biomedcentral.com/1471-2458/14/530

17. Mercer SW, Watt GCM. The inverse care law: clinical primary care encounters in deprived and affluent areas of Scotland. Ann Fam Med. 2007; 5:503-10.

18. Barnett K, Mercer SW, Norbury M, Watt G, Wyke S, Guthrie B. Epidemiology of multimorbidity and implications for health care, research, and medical education: a cross-sectional study. Lancet. 2012;380:37-43.

19. Kunna R, Sebastian MS, Williams JS. Measurement and decomposition of socioeconomic inequality in single and multimorbidity in older adults in China and Ghana: results from the WHO study on global AGEing and adult health (SAGE). Int J Equity Health. 2017;16(1):79.

20. Public Health Agency of Canada. Reducing Health Inequalities: A Challenge For Our Times. 2011. Available from: http://publications.gc.ca/collections/ collection_2012/aspc-phac/HP35-22-2011-eng.pdf.
21. Department of Health. Tackling health inequalities: a programme for action. 2003. Available from: http://webarchive.nationalarchives.gov.uk/ 20031220221853/http://doh.gov.uk/healthinequalities/programmeforaction/ programmeforaction.pdf.

22. Benchimol El, Smeeth L, Guttmann A, Harron K, Moher D, Petersen I, et al. The REporting of studies conducted using observational routinely-collected health data (RECORD) statement. PLoS Med. 2015;12:e1001885-22. Public Library of Science.

23. Béland Y. Canadian community health survey-methodological overview. Health Rep. 2002;13:9-14.

24. Statistics Canada. Canadian Community Dent Health Survey - Annual Component (CCHS). 2018 [cited June 19 2018]. Available from: https://www. statcan.gc.ca/eng/survey/household/32260.

25. Mondor L, Maxwell CJ, Bronskill SE, Gruneir A, Wodchis WP. The relative impact of chronic conditions and multimorbidity on health-related quality of life in Ontario long-stay home care clients. Qual Life Res. 2016;25:2619-32.

26. Mondor L, Maxwell CJ, Hogan DB, Bronskill SE, Gruneir A, Lane NE, et al. Multimorbidity and healthcare utilization among home care clients with dementia in Ontario, Canada: a retrospective analysis of a population-based cohort. PLoS Med. 2017;14:e1002249. Brayne C, editor Public Library of Science

27. Thavorn K, Maxwell CJ, Gruneir A, Bronskill SE, Bai Y, Koné Pefoyo AJ, et al. Effect of socio-demographic factors on the association between multimorbidity and healthcare costs: a population-based, retrospective cohort study. BMJ Open. 2017;7:e017264-15. British Medical Journal Publishing Group

28. Austin PC, Daly PA, Tu JV. A multicenter study of the coding accuracy of hospital discharge administrative data for patients admitted to cardiac care units in Ontario. Am Heart J. 2002;144:290-6. Elsevier

29. Gershon AS, Wang C, Guan J, Vasilevska-Ristovska J, Cicutto L, To T. Identifying patients with physician-diagnosed asthma in health administrative databases. Can Respir J. 2009;16:183-8.

30. Gershon AS, Wang C, Guan J, Vasilevska-Ristovska J, Cicutto L, To T. Identifying individuals with Physcian diagnosed COPD in health administrative databases. COPD. 2009;6:388-94.

31. Schultz SE, Rothwell DM, Chen Z, Tu K. Identifying cases of congestive heart failure from administrative data: a validation study using primary care patient records. Chronic Dis Inj Can. 2013:33:160-6.

32. Jaakkimainen RL, Bronskill SE, Tierney MC, Herrmann N, Green D, Young J, et al. Identification of physician-diagnosed Alzheimer"s disease and related dementias in population-based administrative data: a validation study using family physicians" electronic medical records. J Alzheimers Dis. 2016:54:337-49. IOS Press

33. Hux JE, Ivis F, Flintoft V, Bica A. Diabetes in Ontario: determination of prevalence and incidence using a validated administrative data algorithm. Diabetes Care. 2002;25:512-6.

34. Tu K, Campbell NR, Chen Z-L, Cauch-Dudek KJ, McAlister FA. Accuracy of administrative databases in identifying patients with hypertension. Open Med. 2007:1:e18-26.

35. Diederichs C, Berger K, Bartels DB. The measurement of multiple chronic diseases-a systematic review on existing multimorbidity indices. J Gerontol. 2011;66:301-11

36. Organization for Economic Co-operation and Development. Growing Income Inequality in OECD Countries: What drives it and how can policy tackle it? OECD forum on tackling inequality: Paris, 2011.

37. Organization for Economic Co-operation and Development. Divided We Stand: Why Inequality Keeps Rising: OECD Publishing; 2012. Available from: https://doi.org/10.1787/9789264119536-en.

38. Pickett KE, Wilkinson RG. Income inequality and health: a causal review. Soc Sci Med. 2015;128:316-26. Elsevier Ltd

39. Yeung CW, Thomas S. Income Imputation for the Canadian Community Health Survey. Canada: Statistics Canada, Methodology Branch; 2012.

40. Fortin M, Haggerty J, Almirall J, Bouhali T, Sasseville M, Lemieux M. Lifestyle factors and multimorbidity: a cross sectional study. BMC Public Health. 2014 14:553-8.

41. Wikström K, Lindström J, Harald K, Peltonen M, Laatikainen T. Clinical and lifestyle-related risk factors for incident multimorbidity: 10-year follow-up of Finnish population-based cohorts 1982-2012. Eur J Intern Med. 2015;26:211-6.

42. Kralj B. Measuring Rurality - RIO2008 BASIC: Methodology and Results. Toronto; 2009. [cited 2016 Sep]. https://www.oma.org/wp-content/uploads/ 2008rio-fulltechnicalpaper.pdf. Accessed June 2018. 
43. World Health Organization. Obesity: Preventing and managing the global epidemic - Report of a WHO consultation. Geneva; World Health Organization; 2000. p. 1-268.

44. Glazier RH, Kopp A, Schultz SE, Kiran T, Henry DA. All the right intentions but few of the desired results: lessons on access to primary care from Ontario's patient enrolment models. Healthcare Q. 2012;15:17-21.

45. Glazier RH, Zagorski BM, Rayner J. Comparison of Primary Care Models in Ontario. ICES Investigative Report. Toronto; 2012. p. 1-41. Available from: http://www.ices.on.ca/ /media/Files/Atlases-Reports/2012/Comparison-ofprimary-care-models-in-Ontario/Full\%20report.ashx.

46. Wagstaff A, Paci P, Van Doorslaer E. On the measurement of inequalities in health. Soc Sci Med. 1991;33:545-57.

47. Erreygers G. Correcting the concentration index. J Health Econ. 2009;28: 504-15.

48. van Doorslaer E, Wagstaff A, Lindelow M. Analyzing health equity using household survey data: A Guide to Techniques and Their Implementation. Washington, D.C; 2008. p. 1-234. Available from: http://siteresources. worldbank.org/INTPAH/Resources/Publications/459843-1195594469249/ HealthEquityFINAL.pdf.

49. Wagstaff A, van Doorslaer E, Watanabe N. On decomposing the causes of health sector inequalities with an application to malnutrition inequalities in Vietnam. J Econ. 2003;112:207-23.

50. Yiengprugsawan V, Lim LL, Carmichael GA, Dear KB, Sleigh AC. Decomposing socioeconomic inequality for binary health outcomes: an improved estimation that does not vary by choice of reference group. BMC Res Notes. 2010;3:57-5.

51. Hosseinpoor AR, van Doorslaer E, Speybroeck N, Naghavi M, Mohammad K, Majdzadeh $\mathrm{R}$, et al. Decomposing socioeconomic inequality in infant mortality in Iran. Int J Epidemiol. 2006;35:1211-9.

52. Wagstaff A, Bilger M, Sajaia Z, Lakshin M. Health equity and financial protection. Washington, D.C; 2011. Available from: http://documents. worldbank.org/curated/en/962191468151159187/pdf/ 622580PUB0heal01476B0extop0id018459.pdf.

53. Wagstaff $A$. The bounds of the concentration index when the variable of interest is binary, with an application to immunization inequality. Health Econ. 2005;14:429-32.

54. Mackenbach JP, Kunst AE. Measuring the magnitude of socio-economic inequalities in health: an overview of available measures illustrated with two examples from Europe. Soc Sci Med. 2003;44:757-71.

55. Keppel K, Pamuk E, Lynch J, Carter-Pokras O, Kim Insun, Mays V, et al. Methodological issues in measuring health disparities. Vital Health Stat 2. 2005;141:1-16.

56. Hosseinpoor AR, Bergen N, Mendis S, Harper S, Verdes E, Kunst A, et al. Socioeconomic inequality in the prevalence of noncommunicable diseases in low- and middle-income countries: results from the world health survey. BMC Public Health. 2012;12:474. BioMed Central

57. Ernstsen L, Strand BH, Nilsen SM, Espnes GA, Krokstad S. Trends in absolute and relative educational inequalities in four modifiable ischaemic heart disease risk factors: repeated cross-sectional surveys from the NordTrøndelag health study (HUNT) 1984-2008. BMC Public Health. 2012;12:266. BioMed Central

58. JE-O A. Inequalities in multimorbidity in South Africa. Int J Equity Health. 2013;12:64.

59. Orueta JF, García-Álvarez A, Alonso-Morán E, Vallejo-Torres L, Nuño-Solinis R. Socioeconomic variation in the burden of chronic conditions and health care provision-analyzing administrative individual level data from the Basque Country, Spain. BMC Public Health. 2013;13:870. BioMed Central

60. Dalstra J. Socioeconomic differences in the prevalence of common chronic diseases: an overview of eight European countries. Int J Epidemiol. 2005;34:316-26.

61. Korda RJ, Paige E, Yiengprugsawan V, Latz I, Friel S. Income-related inequalities in chronic conditions, physical functioning and psychological distress among older people in Australia: cross-sectional findings from the 45 and up study. BMC Public Health. 2014;14:741. BioMed Central

62. McGrail KM, van Doorslaer E, Ross NA, Sanmartin C. Income-related health inequalities in Canada and the United States: a decomposition analysis. Am J Public Health. 2009;99:1856-63.

63. van Doorslaer E, Koolman X, Jones AM. Explaining income-related inequalities in doctor utilisation in Europe. Health Econ. 2004;13:629-47.

64. Farrell L, Hollingsworth B, Propper C, Shields MA. The socioeconomic gradient in physical inactivity: evidence from one million adults in England. Soc Sci Med. 2014;123:55-63. Elsevier Ltd
65. Hajizadeh M, Campbell MK, Sarma S. Socioeconomic inequalities in adult obesity risk in Canada: trends and decomposition analyses. Eur J Health Econ. 2014;15:203-21.

66. Beiser M. The health of immigrants and refugees in Canada. Can J Public Health. 2005;96(Suppl 2):S30-44.

67. McDonald JT, Kennedy S. Insights into the "healthy immigrant effect": health status and health service use of immigrants to Canada. Soc Sci Med. 2004; 59:1613-27.

68. Starfield B. Primary care: an increasingly important contributor to effectiveness, equity, and efficiency of health services. SESPAS report 2012 Gac Sanit. 2012;26(Suppl 1):20-6.

69. Aggarwal M, Hutchison B. Toward a primary care strategy for Canada [internet]. 2012. Available from: http://www.cfhi-fcass.ca/Libraries/Reports/ Primary-Care-Strategy-EN.sflb.ashx

70. Starfield B, Shi L, Macinko J. Contribution of primary care to health systems and health. Milbank Q. 2005;83:457-502. Blackwell Publishing, Inc.

\section{Ready to submit your research? Choose BMC and benefit from:}

- fast, convenient online submission

- thorough peer review by experienced researchers in your field

- rapid publication on acceptance

- support for research data, including large and complex data types

- gold Open Access which fosters wider collaboration and increased citations

- maximum visibility for your research: over $100 \mathrm{M}$ website views per year

At BMC, research is always in progress.

Learn more biomedcentral.com/submissions 